\title{
Evolução Clínica e Preditores da Neuropatia Periférica Induzida por Quimioterapia
}

doi: https://doi.org/10.32635/2176-9745.RBC.2019v65n2.392

\author{
Clinical Evolution and Predictors of Chemotherapy-Induced Peripheral Neuropathy \\ Evolución Clínica y Predictores de la Neuropatía Periférica Inducida por Quimioterapia
}

\author{
Delma Aurélia da Silva Simão'; Mery Natali Silva Abreu²; Rodrigo Santiago Gomez³; Leonardo Dornas de Oliveira4; Raissa Silva \\ Souza ${ }^{5}$; Tércia Moreira Ribeiro da Silva ${ }^{6}$; Antonio Lúcio Teixeira ${ }^{7}$
}

\begin{abstract}
Resumo
Introduçáo: Drogas antineoplásicas neurotóxicas estão frequentemente associadas à neuropatia periférica induzida por quimioterapia (NPIQ). Objetivo: Avaliar a evolução clínica dos pacientes expostos a tratamento antineoplásico potencialmente neurotóxico e identificar possíveis preditores clínicos e sociodemográficos para o desenvolvimento da NPIQ. Método: Estudo de coorte prospectiva com pacientes com diagnóstico de câncer de mama, ovário ou intestino em tratamento quimioterápico com paclitaxel, docetaxel ou oxaliplatina. Foram avaliados antes da quimioterapia (T1), no terceiro mês (T2) e 30-60 dias após interrupçáo do tratamento (T3). Todos responderam ao questionário de perfis sociodemográfico e clínico, foram avaliados por meio de exame clínico neurológico, pela escala de performance ECOG, escala hospitalar de ansiedade e depressão (HAD), escala de dor Short-cGuill, autorrelato de sintomas de NPIQ e avaliaçáo com o questionário de neurotoxicidade induzida por antineoplásicos (CINQ). Resultados: Por meio de autorrelato, $75 \%$ da dos pacientes informaram apresentar sintomas de NPIQ. O CINQ evidenciou que 90\% apresentaram algum grau de NPIQ em T2, enquanto 82,5\% ainda persistiam em T3. Dor neuropática acometeu $42 \%$ da população ( $R R=1,429$; IC95\%=1,130-1,806). Os escores de ansiedade e depressão reduziram significativamente quando comparados ao início de tratamento (redução de 2,5 pontos na escala $\mathrm{HAD}, \mathrm{p}<0,05$ ). A capacidade funcional da população não mostrou alteraçóes significativas. No T2, a escolaridade foi considerada preditora para autorrelato de sintomas de NPIQ $(\mathrm{OR}=1,314$, IC95\%=1,002-1,723, $\mathrm{p}=0,048)$. Conclusáo: A baixa escolaridade pode comprometer a capacidade do paciente em relatar os sintomas da NPIQ. Este estudo chama a atenção para a necessidade de utilização de instrumentos específicos para detecção precoce da NPIQ. Palavras-chave: Neoplasias; Doenças do Sistema Nervoso Periférico; Antineoplásicos; Síndromes Neurotóxicas; Tratamento Farmacológico.
\end{abstract}

\begin{abstract}
Introduction: Neurotoxic antineoplastic drugs are frequently associated to chemotherapy-induced peripheral neuropathy (CIPN). Objective: To evaluate the clinical evolution of patients exposed to potentially neurotoxic antineoplastic treatment and to identify possible clinical and sociodemographic predictors for the development of CIPN. Method: Cohort prospective study with patients with breast, ovary or intestine diagnosis of cancer in chemotherapy treatment with paclitaxel, docetaxel or oxaliplatin. They were assessed before the chemotherapy (T1), in the third month (T2) and 30-60 days after the interruption of the treatment (T3). All the patients responded to the questionnaire of clinical and sociodemographic profiles, were evaluated through neurologic clinical exam, by the performance scale ECOG, by the Hospital Anxiety and Depression Scale - HAD, pain scale of Short-cGuill, self-report of symptoms of CIPN and evaluation with the questionnaire of antineoplastic-induced neurotoxicity (QAIN). Results: Through self-report, 75\% of the patients presented symptoms of CIPN. The QAIN showed that $90 \%$ presented a certain degree of CIPN in T2, while $82.5 \%$ still persisted in T3. Neuropathic pain affected $42 \%$ of the population $\left(\mathrm{RR}=1.429, \mathrm{CI}_{95 \%}=1.130-1.806\right)$. Anxiety and depression scores significantly reduced when compared with the beginning of the treatment (reduction of 2.5 points in the scale HAD, $\mathrm{p}<0.05$ ). The functional capacity of the population did not show any significant change. The school level was considered a predictor of self-report of CIPN symptoms in T2 $(\mathrm{OR}=1.314$, $\mathrm{CI}_{95 \%}=1.002-1.723, \mathrm{p}=0.048$ ). Conclusion: The low school level may taint the patient capacity to report CIPN symptoms. This study draws attention for the necessity to use specific instruments for early detection of CIPN.

Key word: Neoplasms; Peripheral Nervous System Diseases; Antineoplastic Agents; Neurotoxicity Syndromes; Drug Therapy.
\end{abstract}

Resumen

Introducción: Los fármacos antineoplásicos neurotóxicos a menudo se asocian con neuropatía periférica inducida por quimioterapia (CIPN). Objetivo: Evaluar la evolución clínica de pacientes expuestos a tratamientos antineoplásicos potencialmente neurotóxicos e identificar posibles predictores clínicos y sociodemográficos para el desarrollo de CIPN. Método: Estudio de cohorte prospectivo con pacientes diagnosticadas con cáncer de mama, ovario o intestino sometidos a quimioterapia con paclitaxel, docetaxel u oxaliplatino. Se evaluaron antes de la quimioterapia (T1), en el tercer mes (T2) y 30-60 días después de la interrupción del tratamiento (T3). Todos respondieron el cuestionario de perfil sociodemográfico y clínico, se evaluaron mediante un examen neurológico clínico, la escala de rendimiento ECOG, la escala de ansiedad y depresión hospitalaria (HAD), la escala de dolor Short-cGuill, el autoinforme de los síntomas de CIPN y la evaluación con el cuestionario de neurotoxicidad inducida por antineoplásicos (CINQ). Resultados: Por autoinforme, el 75\% de la población informó presentar síntomas de CIPN. El CINQ mostró que el $90 \%$ tenía algún grado de NPIQ en T2, mientras que el $82.5 \%$ aún persistía en T3. El dolor neuropático afectó al $42 \%$ de la población $(\mathrm{RR}=1.429$; IC del $95 \%=1.130-1.806$ ). Las puntuaciones de ansiedad y depresión disminuyeron significativamente en comparación con el valor inicial (reducción de 2.5 puntos $\mathrm{HAD}, \mathrm{p}<0.05$ ). La capacidad funcional de la población no mostró cambios significativos. En T2, la educación se consideró un predictor de síntomas CIPN autoinformados $(\mathrm{OR}=1.314$, IC 95\%=1.002-1.723, p=0,048). Conclusión: La baja educación puede comprometer la capacidad del paciente para informar los síntomas de CIPN. Este estudio llama la atención sobre la necesidad de utilizar instrumentos específicos para la detección temprana de CIPN. Palabras clave: Neoplasias; Enfermedades del Sistema Nervioso Periférico; Antineoplásicos; Síndromes de Neurotoxicidad; Tratamiento Farmacológico.

\footnotetext{
${ }^{1}$ Laboratório Interdisciplinar de Investigação Médica da Faculdade de Medicina da Universidade Federal de Minas Gerais (UFMG). Departamento Materno Infantil e Saúde Pública da Escola de Enfermagem da UFMG. Belo Horizonte (MG), Brasil. Orcid iD: https://orcid.org/0000-0003-0961-8213

${ }^{2}$ Departamento de Enfermagem Aplicada da Escola de Enfermagem da UFMG. Belo Horizonte (MG), Brasil. Orcid iD: https://orcid.org/0000-0002-6691-3537

${ }^{3}$ Hospital das Clínicas da UFMG. Ambulatório de Cefaleia e Doenças Neuromusculares. Belo Horizonte (MG), Brasil. Orcid iD: https://orcid.org/0000-0002-2093-8528

${ }^{4}$ Hospital das Clínicas da UFMG. Ambulatório de Neurologia. Belo Horizonte (MG), Brasil. Orcid iD: https://orcid.org/0000-0001-6020-6498

${ }^{5}$ Universidade Federal de São João Del-Rei. Divinópolis (MG), Brasil. Orcid iD: https://orcid.org/0000-0001-5010-763X

${ }^{6}$ Departamento Materno Infantil e Saúde Pública da Escola de Enfermagem da UFMG. Belo Horizonte (MG), Brasil. Orcid iD: https://orcid.org/0000-0002-5261-2266

${ }^{7}$ Laboratório Interdisciplinar de Investigação Médica da Faculdade de Medicina da UFMG. Belo Horizonte (MG), Brasil. Neuropsychiatry Program, Department of Psychiatry and Behavioral Sciences, University of Texas Health Science Center at Houston. Houston, EUA. Orcid iD: https://orcid.org/0000-0002-9621-5422

Endereço para correspondência: Delma Aurélia da Silva Simão. Departamento Materno Infantil e Saúde Pública da Escola de Enfermagem da UFMG. Avenida Alfredo Balena, 190 - Santa Efigênia. Belo Horizonte (MG), Brasil. CEP 30130-100. E-mail: enfdelma@yahoo.com.br
} 


\section{INTRODUÇÃO}

Drogas antineoplásicas neurotóxicas como taxanos, derivados da platina, inibidores de proteossomas e alcaloides da vinca, estáo frequentemente associadas à neuropatia periférica induzida por quimioterapia $(\mathrm{NPIQ})^{1}$. Esta é caracterizada por axoniopatia simétrica bilateral e distal, traduzida por queda na amplitude do potencial de ação e aumento da latência distal. Os nervos sensitivos são os mais precocemente acometidos ${ }^{2}$.

Seus sintomas começam, geralmente, no início do tratamento quimioterápico, entre o primeiro e o terceiro ciclo, e com um pico de gravidade com três meses de terapia ${ }^{3,4}$. Manifestaçóes sensitivas positivas (parestesia, disestesia, dor neuropática) são as mais frequentes e, apesar de não se associarem à maior mortalidade, têm impacto significativo na qualidade de vida das pessoas e influenciam a adesão e a continuidade do tratamento ${ }^{1,4}$.

Existem poucas evidências sobre como os parâmetros clínicos podem influenciar o desenvolvimento da NPIQ ${ }^{4-7}$. Alguns autores descrevem a associação entre as diferentes características do protocolo quimioterápico, tais como tipo de droga neurotóxica, intervalo entre os ciclos, dose e número de ciclos como possíveis preditores clínicos para o seu desenvolvimento ${ }^{4-7}$. Diante disso, o objetivo deste trabalho é avaliar a evoluçáo clínica dos pacientes expostos a tratamento antineoplásico potencialmente neurotóxico e identificar possíveis preditores clínicos e sociodemográficos para o desenvolvimento da NPIQ.

\section{MÉTODO}

Trata-se de um estudo de coorte prospectiva que incluiu pacientes com diagnóstico de câncer de mama, ovário ou intestino, com intenção curativa ou paliativa de tratamento, que iniciariam terapia antineoplásica com taxanos (paclitaxel ou docetaxel) ou oxaliplatina, entre janeiro e outubro de 2012, em uma unidade de assistência de alta complexidade em oncologia de um hospital universitário público localizado em Minas Gerais. O estudo foi avaliado e aprovado pelo Comitê de Ética em Pesquisa da Universidade Federal de Minas Gerais (Coep-UFMG), sob o número de parecer 136.463 de 31/10/2012.

Para o cálculo amostral, estimou-se em $60 \%$ a incidência de NPIQ na população exposta ${ }^{5}$ e, em $10 \%$, a presença de neuropatias periféricas na população não exposta a agentes antineoplásicos. Nesse caso, considerando-se um nível de significância de $5 \%$ e o poder do teste em 95\%, obteve-se uma amostra de 30 indivíduos para compor a população. Entretanto, segundo $\mathrm{Miot}^{8}$, o cálculo amostral de estudos longitudinais deve considerar um percentual em torno de $30 \%$ de perdas de seguimento ${ }^{8}$. Nesse caso, o tamanho da população foi ajustado para 40 indivíduos em seguimento.

Os critérios de inclusão foram: não ter realizado nenhum ciclo de tratamento do antineoplásico neurotóxico até a primeira avaliação, ter idade acima de 18 anos e diagnóstico de câncer com tumor localizado em mama, ovário ou intestino. Foram adotados como critérios de exclusão: presença de limitaçóes cognitivas ou transtornos psiquiátricos; pessoas clinicamente debilitadas com pelo índice de capacidade funcional maior ou igual a três (performance status $=\mathrm{PS} \geq 3$ ); ter diagnóstico de diabetes ou hanseníase; apresentar lesóes ou traumas que limitassem a avaliaçáo de pés ou mãos; ter história de lesão neurológica sensitiva ou motora, decorrentes de doenças neurológicas, tais como metástases cerebrais e sequelas de acidente vascular encefálico; ter diagnóstico de artrite reumatoide, síndrome do túnel do carpo ou outras doenças reumatológicas; uso de antibioticoterapia nas quatro semanas que antecedem a primeira avaliação; uso de anti-inflamatórios não esteroides ou glicocorticoides nas duas semanas que antecedem a primeira avaliação; uso de bebida alcoólica mais de três vezes por semana; ser portador de doença renal, insuficiência de adrenais ou doenças do sistema imune, como lúpus; deficiência de vitamina $\mathrm{B} 12$ não tratada; diagnóstico de neuropatia periférica de qualquer origem antes da quimioterapia.

Os pacientes recrutados foram avaliados em três momentos: primeira avaliação: na semana zero, antes de iniciar o tratamento antineoplásico (T1); segunda avaliação: entre as semanas nove a $12 \mathrm{em}$ caso de protocolos que aconteciam a cada 21 dias (T2). No caso específico de pacientes com câncer de mama que faziam uso do protocolo conhecido como AC-T (adriamicina/ ciclofosfamida, quatro ciclos a cada 21 dias, seguido de 12 sessóes semanais de paclitaxel), a segunda avaliação foi feita entre as semanas seis a nove do tratamento com paclitaxel; terceira avaliação: até 60 dias após término do tratamento ou, em caso de tratamento paliativo, entre semanas 21 a 24 (T3).

Os pacientes recrutados foram submetidos a diferentes avaliaçóes, incluindo os perfis sociodemográfico e clínico a partir da coleta das seguintes variáveis: idade (em anos), escolaridade (em anos), sexo, estado civil, consumo de cigarro, consumo de álcool, tipo de antineoplásico neurotóxico, protocolo antineoplásico, tipo de câncer, estadiamento, metástases, objetivo quimioterápico, comorbidades, uso de medicamento em domicílio.

A mensuração da capacidade funcional foi realizada por meio da escala de performance ECOG, desenvolvida pelo Eastern Cooperative Oncology Group e validada pela Organização Mundial da Saúde (OMS) em 1982. Para 
avaliação de sintomas de ansiedade e depressão, utilizou-se a escala hospitalar de ansiedade e depressão (do inglês, Hospital Anxiety and Depression Scale - HAD) ${ }^{10,11}$. A dor foi avaliada utilizando o questionário de dor McGill, em sua forma reduzida - Short Form McGill Questionnaire (SF-MPQ) ${ }^{12,13}$.

Para a avaliação da NPIQ, adotaram-se as seguintes estratégias: autorrelato de sintomas; exame clínico neurológico e avaliação utilizando o questionário de neurotoxicidade induzida por antineoplásicos (CINQ); um questionário validado para a população brasileira a partir de um estudo desenvolvido por Simáo et al. ${ }^{13}$, que obteve, na análise de concordância, índice Kappa $=0,320$ $(\mathrm{p}<0,001)$ e correlação moderada e positiva $(\mathrm{p}=0,357$; $\mathrm{p}<0,001)$ ao comparar o CINQ ao método do estesiômetro ou monofilamentos de Semmes-Weinstein (MSW).

Durante a entrevista clínica, a primeira pergunta era: "Como o(a) Sr.(a.) tem se sentido com a quimioterapia?", "percebe algum efeito colateral?" "percebe algum desconforto com as mãos, pés ou face?”. As respostas eram então registradas por meio de variável dicotômica: presença ou ausência de autorrelato de sintomas de NPIQ. Em seguida, realizou-se exame clínico neurológico sistematizado. Por fim, aplicava-se o CINQ composto por 29 itens que avaliam 20 sintomas divididos em três subescalas: sintomas de neuropatia aguda e crônica em membros inferiores (nove itens); sintomas de neuropatia aguda e crônica em membros superiores (dez itens) e sintomas orofaciais de neuropatia aguda e crônica (10 itens $)^{6,14}$. Os resultados obtidos no CINQ são classificados em graus de 0 a 4, sendo: valor 0 - ausência de sintoma ou sem parestesias; grau 1 - sintomas de curta duração e que não interferem nas atividades de vida diária; grau 2 - parestesias leves que interferem em algumas funçóes, mas não nas atividades básicas de vida diária; grau 3 dor ou comprometimento funcional que interferem nas atividades de vida diária; e grau 4 - sintomas persistentes incapacitantes.

Testes de normalidade foram realizados, obtendo-se variáveis com distribuição não paramétrica, sendo necessário, portanto, optar por testes não paramétricos para as variáveis discretas. Teste de Friedman para variáveis numéricas e Cochran para variáveis categóricas foram realizados para verificar se ocorreram diferenças significativas ao longo do tempo. Nesse caso, realizaram-se análises comparando T1xT2, T1xT3 e T2xT3 com os testes de Wilcoxon para variáveis numéricas, McNemar para variáveis categóricas dicotômicas e teste de homogeneidade marginal para categóricas dicotômicas ordinais. Para as múltiplas comparaçôes, foi adotado o valor $\mathrm{p}=0,029$ (correção de Bonferroni).

Para a análise de preditores de desenvolvimento da NPIQ, foram realizadas análises comparativas entre as características clínicas e sociodemográficas quanto à presença de NPIQ em cada tempo (T1, T2 e T3) comparando-as aos achados de NPIQ identificados por meio de autorrelatos e do CINQ. Nesse caso, foram utilizados o teste exato de Fisher para variáveis categóricas e o teste Mann-Whitney para variáveis numéricas. Regressão logística binária foi realizada nos T2 e T3. Utilizou-se o teste de Hosmer-Lemeshow com estratégia backward (IC95\%). Foram incluídas no modelo de regressão logística as covariáveis com $\mathrm{p}<0,20$.

\section{RESULTADOS}

Durante 18 meses de recrutamento, 262 pacientes atenderam aos critérios de inclusão do estudo. Após avaliação dos critérios, foi necessária a exclusão de 207 (79\% dos pacientes elegíveis), resultando em uma populaçáo de 55 pacientes para seguimento, sendo que $27,3 \%$ tiveram perda de seguimento por abandono do estudo, logo após a avaliação de linha de base, resultando em uma populaçáo final de 40 pacientes (Figura 1).

A população total $(n=55)$ foi composta por pacientes com idades que variaram de 32 a 80 anos (mediana 54 anos). Houve predomínio do sexo feminino $(83,6 \%)$, em geral eram casados $(45,5 \%)$, e tiveram até três filhos $(67,3 \%)$. O nível de escolaridade foi baixo, uma vez que a

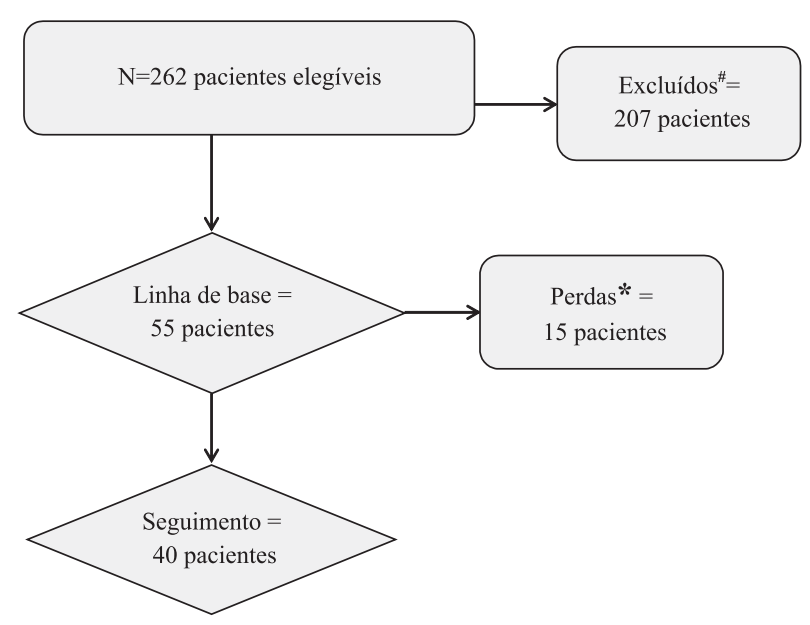

Figura 1. Fluxograma de pacientes elegíveis, excluídos e seguimento na coorte prospectiva de avaliação da NPIQ

Legendas: \# Artrite artrose (7 casos), diabetes (44 casos), dificuldade de locomoçáo (2 casos), doença neurológica ( 4 casos), etilismo crônico (12 casos), início urgente de quimioterapia (26 casos), lesão em MMII com engessamento ( 1 caso), nefropatia ( 1 caso), neuropatia periférica prévia (7 casos), déficit cognitivo importante ( 9 casos), PS $>3$ ( 55 casos), recusa em participar (12 casos), sem telefone de contato (23 casos), tratamento prévio de hanseníase (1 caso), uso de corticoides, anti-inflamatórios não esteroides ou antibióticos até 15 dias antes da avalição (3 casos). *Perda de seguimento por abandono do protocolo de pesquisa em razão da progressāo da doença $(n=7)$, do óbito $(n=3)$, da mudança de protocolo antineoplásico $(n=3)$, de transferência $(n=1)$, do diagnóstico de artrite e artrose pós-avaliação de linha de base (n=1). 
maioria teve menos de 11 anos de estudo e, portanto, não chegaram a completar o ensino médio (72,7\%). A maioria $(50,9 \%)$ se autodenominava católica e informou frequentar a igreja uma vez por semana ou mais. Quanto ao consumo de tabaco e álcool, houve predomínio daqueles que nunca fumaram $(49,1 \%)$ e nunca beberam (45,5\%).

Quanto ao perfil clínico, a maioria iria iniciar tratamento com paclitaxel $(63,6 \%)$, em caráter adjuvante, sendo o câncer de mama predominante na população $(60,4 \%)$. O protocolo ACT, o qual corresponde ao esquema de adriamicina (A) e ciclofofasmida (C) combinadas, sendo quatro ciclos de AC com intervalos de 21 dias, seguidos de 12 semanas consecutivas de paclitaxel $(\mathrm{T})$, seria iniciado em $45,5 \%(\mathrm{n}=25)$ dos pacientes. A presença de outras comorbidades foi identificada em 74,1\% ( $n=41)$ dos pacientes, com destaque para hipertensão arterial sistêmica. A capacidade funcional predominante foi o índice zero de ECOG, em que o paciente é completamente ativo, sem evidência de sintomas da doença oncológica (56,4\%).

Quadros de depressão ou ansiedade foram considerados improváveis para a maioria da populaçáo. A presença de dor de diferentes tipos, exceto dor neuropática, sete dias antes da primeira avaliação, foi constatada em $60 \%$ da população, sendo localizada na região tumoral, associada ou não a dores ósseas, articulares, musculares e cefaleia.

Em análise comparativa realizada entre o grupo seguimento e o grupo perda, não foram encontradas diferenças estatisticamente significativas para as variáveis sociodemográficas. Contudo, nas variáveis clínicas, os pacientes do grupo perda apresentavam pior capacidade funcional de acordo com a escala de performance ECOG $(\mathrm{p}=0,002)$ e sentiam dor com maior frequência $(\mathrm{p}=0,013)$ quando comparados ao grupo seguimento.

As características sociodemográficas e clínicas da população seguimento $(\mathrm{n}=40)$ estão detalhadas na Tabela 1. Houve predomínio de pacientes do sexo feminino ( $\mathrm{n}=32,80 \%)$, com nível de escolaridade baixo e índice zero de capacidade funcional ECOG, em que o sujeito é completamente ativo $(67,5 \%)$. Nota-se que a maioria iria iniciar tratamento com paclitaxel (55\%), em caráter adjuvante, sendo o câncer de mama o predominante na população $(60,4 \%)$. Transtornos de depressão ou ansiedade foram considerados pouco prováveis para a maioria da população.

A análise da evolução clínica incluiu capacidade funcional, dor, sintomas de ansiedade e depressão, autorrelato de NPIQ e avaliaçáo da NPIQ com o CINQ (Tabelas 2, 3 e 4). Os resultados do exame clínico neurológico não apontaram para alterações clínicas significativas ao longo do tempo, exceto pela redução da percepção do reflexo bicipital direito $(\mathrm{T} 1 \mathrm{xT} 3 \mathrm{p}=0,020)$.
Contudo, por meio do autorrelato, constatou-se que, tanto em T2 como em T3, 75\% (n=30) da população informaram apresentar sintomas de NPIQ (Tabela 2). O CINQ, por sua vez, evidenciou que 90\% $(n=36)$ apresentaram algum grau de NPIQ em T2, enquanto $82,5 \%$ $(\mathrm{n}=33)$ ainda persistiam com algum grau da síndrome em T3, independentemente do tipo de antineoplásico (Tabela 2). Apesar de três $(7,5 \%)$ pacientes terem melhorado do quadro de NPIQ após o término do tratamento, percebe-se que, nos demais, houve aumento nos escores dos sintomas elencados nas subescalas para membros inferiores e superiores do CINQ, o que reflete o aumento na intensidade e na frequência dos sintomas em membros inferiores e superiores com consequente e progressivo impacto nas atividades de vida diária, mesmo após a interrupção da exposição ao antineoplásico (Tabela 2).

Observou-se também que, ao longo do tratamento, foi identificado aumento significativo da presença de dor neuropática (Tabela 2). Análises identificaram que, apesar de a dor de outros tipos náo ter progredido ao longo do tratamento, 17 (42\%) pacientes desenvolveram dor neuropática em algum momento durante o estudo. Em T2, foi constatado um risco de $43 \%$ para desenvolvimento de dor neuropática em pacientes que apresentavam algum sintoma de NPIQ (RR=1,429; IC95\%=1,130-1,806). Não houve alteraçôes significativas dos quadros de dor neuropática entre T2 e T3, o que evidencia também um caráter persistente do quadro.

Os escores de ansiedade e depressão reduziram significativamente quando comparados ao início de tratamento. A capacidade funcional da população avaliada pelo índice ECOG não mostrou alterações significativas (Tabela 2).

No T2, a escolaridade foi a única variável considerada preditora de autorrelato de sintomas de NPIQ $(\mathrm{OR}=1,314$, IC95\%=1,002- 1,723, $\mathrm{p}=0,048$ ) (Tabela 3). Nesse modelo de regressão, foram incluídas as variáveis: gênero, situação de trabalho, escolaridade, peso, número de doses do antineoplásico, tempo de diagnóstico, uso de medicamentos em casa, capacidade funcional, presença de dor e intensidade e da dor (Tabela 3).

Observou-se associação entre a síndrome identificada pelo CINQ e o escore de depressão no T3 ( $\mathrm{p}=0,018)$ (Tabela 3). Não houve relação entre NPIQ com diferentes características do protocolo adotado, tais como tipo de droga neurotóxica, intervalo entre os ciclos, dose e número de ciclos. No modelo de regressão realizado, foram incluídas as variáveis gênero, situação de trabalho, peso, estadiamento, uso de medicamentos em casa, capacidade funcional, escore de depressão, presença de dor e intensidade da dor (Tabela 4). Contudo, nenhuma das covariáveis permaneceu no modelo, o que sugere que, 
Tabela 1. Características sociodemográficas e clínicas da população de pacientes com câncer antes do início do tratamento antineoplásico potencialmente neurotóxico $(n=40)$

\begin{tabular}{|c|c|c|}
\hline Parâmetros & & Seguimento $(n=40) n(\%)$ \\
\hline Idade (em anos) [mediana (P25-P75)] & & $53,5(33-80)$ \\
\hline Escolaridade (em anos) [mediana (P25-P75)] & & $8(2-20)$ \\
\hline \multirow[t]{2}{*}{ Sexo } & Feminino & $32(80,0 \%)$ \\
\hline & Masculino & $8(20,0 \%)$ \\
\hline \multirow[t]{3}{*}{ Estado civil } & Solteiro & $7(17,5 \%)$ \\
\hline & Casado & $20(50,0 \%)$ \\
\hline & Separado & $8(20,0 \%)$ \\
\hline \multirow[t]{3}{*}{ Consumo de cigarro } & Nunca fumou & $21(52,5 \%)$ \\
\hline & Fumou no passado & $13(32,5 \%)$ \\
\hline & Fuma atualmente & $6(15,0 \%)$ \\
\hline \multirow[t]{3}{*}{ Consumo de álcool } & Nunca bebeu & $19(47,5 \%)$ \\
\hline & Bebeu no passado & $15(37,5 \%)$ \\
\hline & Bebe atualmente & $6(15,0 \%)$ \\
\hline \multirow[t]{3}{*}{ Tipo de antineoplásico neurotóxico } & Paclitaxel & $24(60,0 \%)$ \\
\hline & Oxaliplatina & $13(32,5 \%)$ \\
\hline & Docetaxel & $3(7,5 \%)$ \\
\hline \multirow[t]{5}{*}{ Protocolo antineoplásico } & $\mathrm{AC}-\mathrm{T}^{\mathrm{a}}$ & $19(47,5 \%)$ \\
\hline & Carbo/paclitaxel $\left.\right|^{b}$ & $3(7,5 \%)$ \\
\hline & b-FOLc ou FLOXd & $8(20,0 \%)$ \\
\hline & Docetaxel/CTXe & $2(5,0 \%)$ \\
\hline & Outros esquemas & $2(5,0 \%)$ \\
\hline \multirow[t]{3}{*}{ Tipo de câncer } & Mama & $24(60,0 \%)$ \\
\hline & Colón/reto & $13(34,2 \%)$ \\
\hline & Outros & $1(2,6 \%)$ \\
\hline \multirow[t]{3}{*}{ Estadiamento } & II & $10(25,0 \%)$ \\
\hline & III & $17(42,5 \%)$ \\
\hline & IV & $13(32,5 \%)$ \\
\hline \multirow[t]{2}{*}{ Metástase } & Presente & $13(32,5 \%)$ \\
\hline & Ausente & $27(67,5 \%)$ \\
\hline \multirow[t]{3}{*}{ Objetivo quimioterápico } & Adjuvante & $20(50,0 \%)$ \\
\hline & Paliativa & $10(25,0 \%)$ \\
\hline & Neoadjuvante & $10(25,0 \%)$ \\
\hline \multirow[t]{2}{*}{ Comorbidades } & Presente & $26(65,0 \%)$ \\
\hline & Ausente & $14(35,0 \%)$ \\
\hline \multirow[t]{2}{*}{ Uso de medicamentos em domicílio } & Sim & $26(65,0 \%)$ \\
\hline & Não & $14(35,0 \%)$ \\
\hline \multirow[t]{3}{*}{ Índice de desempenho diário (PS ECOG 0-4) } & 0 & $27(67,5 \%)$ \\
\hline & 1 & $11(27,5 \%)$ \\
\hline & 2 & $2(5,0 \%)$ \\
\hline \multirow[t]{2}{*}{ Ansiedade (escala HAD) } & Pouco provável & $33(82,5 \%)$ \\
\hline & Provável & $7(17,5 \%$ \\
\hline \multirow{2}{*}{ Depressão (escala HAD) } & Pouco provável & $32(80,0 \%)$ \\
\hline & Provável & $8(20,0 \%)$ \\
\hline \multirow[t]{2}{*}{ Dor } & Presente & $20(50,0 \%)$ \\
\hline & Ausente & $20(50,0 \%)$ \\
\hline \multirow[t]{3}{*}{ Intensidade da dor } & Leve & $8(20,0 \%)$ \\
\hline & Moderada & $9(22,5 \%)$ \\
\hline & Intensa & 3 (7,5\%) \\
\hline
\end{tabular}

Legendas: a AC-T: adriamicina $60 \mathrm{mg} / \mathrm{m}^{2} /$ ciclofosfamida $600 \mathrm{mg} / \mathrm{m}^{2}$, por 4 ciclos, a cada 21 dias, seguido de paclitaxel $80 \mathrm{mg} / \mathrm{m}^{2}$, em regime semanal durante 12 semanas (sendo 1 ciclo a cada 3 doses). ${ }^{b}$ Carbo/Taxol: carboplatina AUC 6/paclitaxel $175 \mathrm{mg} / \mathrm{m}^{2}$ a cada 3 semanas. ${ }^{c}$ b-FOL: oxaliplatina $85 \mathrm{mg} / \mathrm{m}$ D1 e D15/ácido

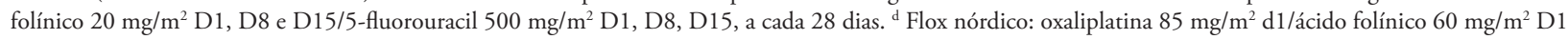
e D2/5- fluorouracil $500 \mathrm{mg} / \mathrm{m}^{2} \mathrm{D} 1{ }^{\mathrm{c}} \mathrm{D} 2$, intervalo a cada 15 dias. e Docetaxel/CTX: docetaxel $75 \mathrm{mg} / \mathrm{m}^{2} /$ ciclofosfamida $600 \mathrm{mg} / \mathrm{m}^{2}$, a cada 21 dias por 4 ciclos. 
Tabela 2. Evolução clínica de pacientes em uso de drogas neurotóxicas $(n=40)$

\begin{tabular}{|c|c|c|c|c|c|c|c|c|}
\hline Parâmetros & & $\begin{array}{c}\mathrm{Tl}(\mathrm{n}=40) \\
\mathrm{N}(\%)\end{array}$ & $\begin{array}{c}\text { T2 }(n=40) \\
N(\%)\end{array}$ & $\begin{array}{c}\text { T3 }(n=40) \\
N(\%)\end{array}$ & $\begin{array}{l}\text { Valor } p \\
(<0,05) \\
\text { T1xT2xT3 }\end{array}$ & $\begin{array}{c}\text { Valor } \mathrm{p}^{*} \\
(<0,029)^{*} \\
\text { TlxT2 }\end{array}$ & $\begin{array}{c}\text { Valor } \mathrm{p}^{*} \\
(<0,029) \\
\text { TlxT3 }\end{array}$ & $\begin{array}{c}\text { Valor } \mathrm{p}^{*} \\
(<0,029) \\
\mathrm{T} 2 \mathrm{xT3}\end{array}$ \\
\hline \multirow{3}{*}{$\begin{array}{l}\text { Índice de desempenho } \\
\text { diário (performance ECOG) }\end{array}$} & 0 & $27(67,5 \%)$ & $21(52,5 \%)$ & $20(64,5 \%)$ & $0,262^{\# \#}$ & - & - & - \\
\hline & 1 & $11(27,5 \%)$ & $17(42.5 \%)$ & $8(25,8 \%)$ & & & & \\
\hline & 2 & $2(05,0 \%)$ & $1(2,5 \%)$ & $3(9,7 \%)$ & & & & \\
\hline \multirow{2}{*}{ Dor neuropática } & Presente & $0(0,0 \%)$ & $09(22,5 \%)$ & $15(37,5 \%)$ & $<0,001^{* *}$ & $0,004^{\dagger}$ & $<0,001^{\dagger}$ & $0,109^{\dagger}$ \\
\hline & Ausente & $40(100,0 \%)$ & $31(77,5 \%)$ & $25(62,5 \%)$ & & & & \\
\hline \multirow{2}{*}{ Dor (todos os tipos) } & Índice de dor total (0-45) & 0 & 0 & 0 & $0,308^{\#}$ & - & - & - \\
\hline & Mediana (min.-máx.) & $(0-33)$ & $(0-28)$ & $(0-26)$ & & & & \\
\hline \multirow{2}{*}{$\begin{array}{l}\text { Intensidade da dor } \\
\text { presente }\end{array}$} & $\operatorname{EVA}(0-10)$ & 0 & 0 & 0 & $0,868^{\#}$ & - & - & - \\
\hline & Mediana (min.-máx.) & $(0-10)$ & $(0-10)$ & $(0-8)$ & & & & \\
\hline \multirow{6}{*}{$\begin{array}{l}\text { Ansiedade e depressão } \\
\text { (Escala HAD) }\end{array}$} & Escore total (0-42) & 9 & 6 & 6,5 & $0,008^{\#}$ & $0,137=$ & 0,009 & 0,057 \\
\hline & Mediana (min.-máx.) & $(1-25)$ & $(0-36)$ & $(0-25)$ & & & & \\
\hline & Escore ansiedade $(0-21)$ & 5 & 3 & 3,5 & $0,001^{\#}$ & 0,070 & 0,012 & $0,126=$ \\
\hline & Mediana (min.-máx.) & $(0-13)$ & $(0-15)$ & $(0-14)$ & & & & \\
\hline & Escore depressão (0-21) & 4 & 3 & 3 & $0,210^{\#}$ & - & - & - \\
\hline & Mediana (min.-máx.) & $(0-15)$ & $(0-14)$ & $(0-13)$ & & & & \\
\hline \multirow{2}{*}{$\begin{array}{l}\text { Sintomas autorrelatados } \\
\text { de NPIQ }\end{array}$} & Presente & $2(05,0 \%)$ & $30(75,0 \%)$ & $30(75,0 \%)$ & $<0,001^{* *}$ & $<, 001^{\dagger}$ & $<0,001^{\dagger}$ & $1,000^{\dagger}$ \\
\hline & Ausente & $38(95,0 \%)$ & $10(25,0 \%)$ & $10(25,0 \%)$ & & & & \\
\hline $\begin{array}{l}\text { CINQ escore total (0-190) } \\
\text { Mediana (min.-máx.) }\end{array}$ & & $3(0-90)$ & $14(0-91)$ & $18,5(0-78)$ & $<0,001$ & $<0,001$ & $<0,001$ & 0,249 \\
\hline $\begin{array}{l}\text { CINQ escore subescala para } \\
\text { membros inferiores (0-90) } \\
\text { Mediana (min.-máx.) }\end{array}$ & & $0(0-45)$ & $6(0-48)$ & $9,5(0-41)$ & $<0,001$ & 0,001 & $<0,001$ & 0,095 \\
\hline $\begin{array}{l}\text { CINQ escore subescala para } \\
\text { membros superiores } \\
\text { Mediana (min.-máx.) }\end{array}$ & & $0(0-22)$ & $3(0-32)$ & $2(0-46)$ & $<0,001$ & $<0,001$ & $<0,001$ & 0,746 \\
\hline $\begin{array}{l}\text { CINQ escore subescala } \\
\text { orofacial (0-100) } \\
\text { Mediana (min.-máx.) }\end{array}$ & & $0(0-40)$ & $3(0-34)$ & $2(0-42)$ & 0,097 & - & - & - \\
\hline
\end{tabular}

Legendas: *Valor p<0,029 (correção de Bonferroni); ${ }^{* *}$ Teste de Cochran; ${ }^{\# T e s t e ~ d e ~ F r i e d m a n ; ~}{ }^{\# \# ~ T e s t e ~ d e ~ K e n d a l l ; ~ † T e s t e ~ d e ~ M c N e m a r ; ~ “ ~ T e s t e ~ d e ~ W i l c o x o n . ~}$

ao utilizar o instrumento apropriado, variáveis clínicas e sociodemográficas não influenciaram a presença de sintomas de NPIQ na população avaliada.

\section{DISCUSSÃO}

Os resultados dessa pesquisa confirmam que a NPIQ é um problema incidente em pacientes expostos a taxanos e oxaliplatina tanto durante quanto após o tratamento, como já evidenciado em outros estudos ${ }^{1,3,7}$.

A maior incidência de sintomas de NPIQ ocorreram no T2 de avaliação, confirmando estudos anteriores que mostraram pico dos sintomas de neuropatia por volta do terceiro ciclo de tratamento ${ }^{4}$. Os sintomas de NPIQ mantiveram estáveis entre as semanas seis e 12, sem haver redução significativa pelo menos até 30 dias após o término da quimioterapia, o que estaria alinhado com o caráter crônico dos quadros neuropáticos ${ }^{6}$. Os resultados apontam também que os sintomas da NPIQ geralmente se iniciam em membros inferiores, tornam-se mais graves e persistem por mais tempo, comparados aos sintomas de membros superiores. Os sintomas orofaciais não evoluíram significativamente na população avaliada.

Destaca-se, nos achados desse trabalho, a não determinação de associação entre diferentes características do protocolo adotado, tais como tipo de droga neurotóxica, intervalo entre os ciclos, dose e número de ciclos, preditores frequentemente citados por diferentes autores ${ }^{4,7,14}$. No presente estudo, a presença de NPIQ decorreu exclusivamente da exposição da pessoa à droga neurotóxica, sendo esta considerada fator de risco iminente para a síndrome. 
Tabela 3. Possíveis preditores sociodemográficos e clínicos para o desenvolvimento da NPIQ segundo QNIA e autorrelato no tempo 2 (T2) e tempo 3 (T3)

\begin{tabular}{|c|c|c|c|c|}
\hline \multirow{2}{*}{ Parâmetros } & \multicolumn{2}{|c|}{$\begin{array}{c}\text { QNIA } \\
\text { (valor } p<0,05)\end{array}$} & \multicolumn{2}{|c|}{$\begin{array}{c}\text { Autorrelato } \\
(\text { valor } p<0,05)\end{array}$} \\
\hline & $\begin{array}{c}\text { T2 } \\
(n=40)\end{array}$ & $\begin{array}{c}\text { T3 } \\
(n=40)\end{array}$ & $\begin{array}{c}\text { T2 } \\
(n=40)\end{array}$ & $\begin{array}{c}\text { T3 } \\
(n=40)\end{array}$ \\
\hline Idade & $0,551^{*}$ & $0,225^{*}$ & $0,866^{*}$ & $0,724 *$ \\
\hline Gênero & $0,563^{\#}$ & $0,172^{\#}$ & $0,361^{\#}$ & $0,068^{\dagger}$ \\
\hline Escolaridade (em anos) & $0,843^{*}$ & $0,983^{*}$ & $0,033^{*}$ & $0,301^{*}$ \\
\hline Estado civil & $0,525^{\#}$ & $0,884^{\#}$ & $0,465^{\dagger}$ & $0,325^{\#}$ \\
\hline Consumo de cigarro & $0,326^{\#}$ & $0,257^{\#}$ & $0,890^{\#}$ & $0,684^{\#}$ \\
\hline Consumo de álcool & $1,000^{\#}$ & $0,635^{\#}$ & $0,888^{\#}$ & $0,534^{\#}$ \\
\hline Tipo de antineoplásico neurotóxico & $0,760^{\#}$ & $0,712^{\#}$ & $0,747^{\#}$ & $0,633^{\#}$ \\
\hline Protocolo antineoplásico & $1,000^{\#}$ & $0,539^{\#}$ & $0,588^{\#}$ & $0,588^{\#}$ \\
\hline Número de doses & $0,589 *$ & $0,712^{*}$ & $0,187^{*}$ & $0,860 *$ \\
\hline Número de ciclos & $0,627^{*}$ & $0,744^{*}$ & $0,724^{*}$ & $0,820^{*}$ \\
\hline Tipo de câncer & $0,692^{\#}$ & $0,630^{\#}$ & $0,778^{\#}$ & $0,778^{\#}$ \\
\hline Estadiamento & $0,186^{\#}$ & $0,437^{\#}$ & $0,338^{\#}$ & $1,000^{\#}$ \\
\hline Metástases & $1,000^{\#}$ & $1,000^{\#}$ & $0,845^{\dagger}$ & $0,845^{\#}$ \\
\hline Comorbidades & $1,000^{\#}$ & $1,000^{\dagger}$ & $0,251^{\dagger}$ & $0,702^{\dagger}$ \\
\hline Uso de medicamentos em domicílio & $0,023^{\#}$ & $0,631^{\dagger}$ & $0,908^{\dagger}$ & $0,088^{\dagger}$ \\
\hline Índice de desempenho diário (PS ECOG 0-4) & $0,437^{\dagger}$ & $0,142^{\#}$ & $0,336^{\#}$ & $0,086^{\dagger}$ \\
\hline Ansiedade (Escala HAD) & $0,228^{*}$ & $0,811^{*}$ & $1,000^{*}$ & $0,363^{*}$ \\
\hline Depressão (Escala HAD) & $0,018^{*}$ & $0,083^{*}$ & $1,000^{*}$ & $0,988^{*}$ \\
\hline Dor & $0,061^{\#}$ & $0,114^{\#}$ & $0,271^{\dagger}$ & 0,097 \\
\hline Intensidade da dor & $0,449 *$ & $0,112^{*}$ & $0,469 *$ & $0,145^{*}$ \\
\hline
\end{tabular}

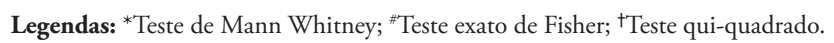

Tabela 4. Graus de neuropatia periférica detectados pelo CINQ em pacientes expostos a tratamento antineoplásico potencialmente neurotóxico nos diferentes tempos $(n=40)$

\begin{tabular}{|c|c|c|c|c|c|c|c|c|c|c|c|}
\hline Parâmetros & & \multicolumn{2}{|c|}{$\begin{array}{c}\text { T1 }(n=40) \\
n \%\end{array}$} & \multicolumn{2}{|c|}{$\begin{array}{c}\text { T2 }(n=40) \\
n \%\end{array}$} & \multicolumn{2}{|c|}{$\begin{array}{c}\text { T3 }(n=40) \\
n \%\end{array}$} & $\begin{array}{c}\text { Valor } p^{\#} \\
(<0,05) \\
\text { T1xT2xT3 }\end{array}$ & $\begin{array}{c}\text { Valor } p^{\dagger} \\
(<0,029) \\
\text { T1 x T2 }\end{array}$ & $\begin{array}{c}\text { Valor } p^{\dagger} \\
(<0,029) \\
\text { T1x T3 }\end{array}$ & $\begin{array}{c}\text { Valor } p^{\dagger} \\
(<0,029) \\
\text { T2x T3 }\end{array}$ \\
\hline \multirow{5}{*}{$\begin{array}{l}\text { Graus de } \\
\text { neuropatia }\end{array}$} & 0 & 16 & 40,0 & 04 & 10,0 & 07 & 17,5 & & & & \\
\hline & 1 & 11 & 27,5 & 16 & 40,0 & 11 & 27,5 & & & & \\
\hline & 2 & 03 & 07,5 & 07 & 17,5 & 08 & 20,0 & & & & \\
\hline & 3 & 04 & 10,0 & 05 & 12,5 & 07 & 17,5 & & & & \\
\hline & 4 & 05 & 12,5 & 08 & 20,0 & 07 & 17,5 & & & & \\
\hline
\end{tabular}

Legendas: *Valor p <0,029 (correção de Bonferroni); "Teste de Kendall; ${ }^{\dagger}$ Teste de homogeneidade marginal.

Entre as características sociodemográficas, apenas a escolaridade foi preditora de sintomas autorrelatados de NPIQ, uma vez que quanto menor a escolaridade menor a chance de o paciente relatar espontaneamente sintomas de NPIQ. A prevalência de pacientes recrutados neste estudo com tempo médio de estudo de oito anos se aproxima daquele perfil identificado em estudos que apontam a expansão do acesso da população de baixa escolaridade aos serviços da atençấo oncológica e do Sistema Único de Saúde no Brasil, especialmente entre os anos de 1998 a $2013^{15,16}$. Nesse contexto, os achados deste estudo reforçam a necessidade de intervençóes mais claras e objetivas, utilizando instrumentos específicos e objetivos que detectem precocemente os sintomas da NPIQ em grupos populacionais com baixa escolaridade, prevenindo assim danos neurais graves decorrentes da terapêutica antineoplásica.

O rigor na adoção dos critérios de exclusão da populaçấo neste estudo pode, de um lado, ter evitado a inclusão de pessoas com quadros neuropáticos de outras 
origens que pudessem influenciar nossos resultados, mas, por outro, limitou também a população para seguimento a 55 indivíduos, entre os quais, $27 \%$ foram considerados perdas. Recente metanálise que avaliou os possíveis preditores clínicos para NPIQ evidenciou a falta de uniformidade dos métodos de avaliaçáo da dor além de problemas relacionados ao tamanho amostral, o que pode dificultar a comparação deste estudo com outros estudos epidemiológicos realizados anteriormente ${ }^{17}$.

Este foi o primeiro estudo epidemiológico, nacional, unicêntrico avaliando a NPIQ em adultos, ao contrário de estudos internacionais que são, em geral, multicêntricos.

\section{CONCLUSÃO}

Em conclusão, a NPIQ é um problema prevalente em pacientes expostos a taxanos e oxaliplatina tanto durante quanto após a terapêutica com maior incidência entre seis e nove semanas de tratamento antineoplásico. A escolaridade de pacientes tratados em unidade ambulatorial de hospital público no Brasil mostrou ser preditora de sintomas autoerrelatados de NPIQ, uma vez que se evidenciou que quanto menor a escolaridade menor é a chance de o paciente relatar espontaneamente sintomas de NPIQ, o que pode sugerir a necessidade de implementação de instrumentos específicos objetivos validados para tal avaliação.

\section{CONTRIBUIÇÕES}

Delma Aurélia da Silva Simáo contribuiu na concepção e planejamento do estudo, obtenção, análise e interpretação dos dados, redação do manuscrito e aprovação final da versão publicada. Mery Natali Silva Abreu contribuiu na concepção e planejamento do estudo, análise e interpretação dos dados, revisão crítica e aprovação final da versão publicada. Rodrigo Santiago Gomez contribuiu na concepçâo e planejamento do estudo, obtenção dos dados, revisão crítica e aprovação final da versão publicada. Leonardo Dornas de Oliveira e Raissa Silva Souza contribuíram no planejamento do estudo, obtenção dos dados, revisão crítica e aprovação final da versão publicada. Tércia Moreira Ribeiro da Silva contribuiu na interpretaçáo dos dados da pesquisa, revisão crítica e aprovação final da versão publicada. Antônio Lúcio Teixeira contribuiu no planejamento do estudo, análise e interpretação dos dados, revisão crítica e aprovação final da versão publicada.

\section{DECLARAÇÃO DE CONFLITO DE INTERESSES}

Nada a declarar.

\section{FONTES DE FINANCIAMENTO}

Fundação de Amparo à Pesquisa de Minas Gerais (Fapemig).

\section{REFERÊNCIAS}

1. Fu X, Wu H, Li J, et al. Efficacy of drug interventions for chemotherapy-induced chronic peripheral neurotoxicity: a network meta-analysis Front Neurol. 2017;8:223. doi: https://doi.org/10.3389/fneur.2017.00223.

2. Simão DAS, Murad M, Martins C, et al. Chemotherapyinduced peripheral neuropathy: review for clinical practice. Rev Dor. 2015;16(3):215-20. doi: https://doi. org/10.5935/1806-0013.20150043.

3. Avan A, Postma TJ, Ceresa C, et al. Platinum-induced neurotoxicity and preventive strategies: past, present, and future. Oncologist. 2015 Apr;20(4):411-432. doi: https://doi.org/10.1634/theoncologist.2014-0044.

4. Pachman DR, Qin R, Seisler D, et al. Comparison of oxaliplatin and paclitaxel-induced neuropathy (Alliance A151505). Support Care Cancer. 2016;24(12):5059-68. doi: https://doi.org/10.1007/s00520-016-3373-1.

5. Cavaletti G, Frigeni B, Lanzani F, et al. The total neuropathy score as an assessment tool for grading the course of chemotherapy-induced peripheral neurotoxicity: comparison with the National Cancer Institute-common toxicity scale. J Peripher Nery Syst. 2007;12(3):210-5. doi: https://doi.org/10.1111/j.15298027.2007.00141.x.

6. Leonard GD, Wright MA, Quinn MG, et al. Survey of oxaliplatin-associated neurotoxicity using an interviewbased questionnaire in patients with metastatic colorectal cancer. BMC Cancer. 2005;5:116:1-10. doi: https://doi. org/10.1186/1471-2407-5-116.

7. Stone JB, DeAngelis LM. Cancer-treatment-induced neurotoxicity--focus on newer treatments. Nat Rev Clin Oncol. 2016 Feb;13(2):92-105. doi: https://doi. org/10.1038/nrclinonc.2015.152.

8. Miot HA. Tamanho da amostra em estudos clínicos e experimentais. J Vasc Bras. 2011;10(4):275278. doi: http://dx.doi.org/10.1590/S167754492011000400001.

9. Stern AF. The hospital anxiety and depression scale. Occup Med (Lond). 2014;64(5):393-4. doi: https://doi. org/10.1093/occmed/kqu024.

10. Turk DC, Dworkin RH, Trudeau JJ, et al. Validation of the hospital anxiety and depression scale in patients with acute low back pain. J Pain. 2015 Oct;16(10):1012-21. doi: https://doi.org/10.1016/j.jpain.2015.07.001.

11. Melzack R. The short-form McGill Pain questionnaire. Pain. 1987 Aug;30(2):191-7. doi: https://doi. org/10.1016/0304-3959(87)91074-8. 
12. Pimenta CAM, Teixeira MJ. Adaptation of McGill questionnaire to portuguese language. Rev Esc Enf USP. 1996;30(3):473-83. doi: http://dx.doi.org/10.1590/ S0080-62341996000300009.

13. Simão DAS, Teixeira AL, Souza RS, et al. Evaluation of the Semmes-Weinstein filaments and a questionnaire to assess chemotherapy-induced peripheral neuropathy. Support Care Cancer. 2014;22(10):2767-73. doi: http:// dx.doi.org/10.1007/s00520-014-2275-3.

14. Banach M, Juranek JK, Zygulska AL. Chemotherapyinduced neuropathies-a growing problem for patients and health care providers. Brain Behav. 2016;7(1):e00558. doi: http://dx.doi.org/10.1002/brb3.558.

15. Viacava F, Oliveira RAD, Carvalho CC, et al. SUS: oferta, acesso e utilização de serviços de saúde nos últimos 30 anos. Cien Saude Colet. 2018;23(6):1751-62. doi: https://doi.org/10.1590/1413-81232018236.06022018.

16. Viacava F, Porto SM, Carvalho CC, et al. Desigualdades regionais e sociais em saúde segundo inquéritos domiciliares (Brasil, 1998-2013). Cien Saude Colet [Internet]. 2018 Jan 9 [acesso 2019 ago. 9]; Ahead of print 49-0002/2018. Disponível em: http://www. cienciaesaudecoletiva.com.br/artigos/desigualdadesregionais-e-sociais-em-saude-segundo-inqueritosdomiciliares-brasil-9982013/16535? id=16535\& $\mathrm{id}=16535$.

17. Seretny M, Currie GL, Sena ES, et al. Incidence, prevalence, and predictors of chemotherapy-induced peripheral neuropathy: a systematic review and metaanalysis. Pain. 2014;155(12):2461-70. doi: http://dx.doi. org/10.1016/j.pain.2014.09.020. 